\title{
Erratum to: Oral intake of a combination of glucosyl hesperidin and caffeine elicits an antiobesity effect in healthy, moderately obese subjects: a randomized double-blind placebo-controlled trial
}

Tatsuya Ohara*, Koutarou Muroyama, Yoshihiro Yamamoto and Shinji Murosaki

\section{Erratum}

After publication of this article [1] it was noticed there were errors in the paper regarding the dose and grade of glucosyl hesperidin due to miscommunications.

The composition of glucosyl hesperidin is reported as "500 mg" but this should instead be listed as " $470 \mathrm{mg}$ " throughout the article. Additionally, the G-hesperidin should be reported as containing $74 \%$ monoglucosyl hesperidin and residual non-glycosylated hesperidin.

Received: 10 May 2017 Accepted: 10 May 2017

Published online: 12 May 2017

\section{Reference}

1. Ohara T, Muroyama K, Yamamoto Y, Murosaki S. Oral intake of a

combination of glucosyl hesperidin and caffeine elicits an antiobesity effect in healthy, moderately obese subjects: a randomized double-blind placebocontrolled trial. Nutr J. 2016;15:6.

* Correspondence: Ohara_Tatsuya@house-wf.co.jp

Research \& Development Institute, House Wellness Foods Corporation, 3-20 Imoji, Itami, Hyogo 664-0011, Japan 